\title{
A Three-Axis Force Fingertip Sensor Based on Fiber Bragg Grating
}

\author{
Yongxing Guo ${ }^{\text {a, }}$, Jianyi Kong ${ }^{\text {a }}$, Honghai Liu ${ }^{\text {a, b }}$, Hegen Xiong ${ }^{\text {a }}$, Gongfa $\mathrm{Li}^{\text {a }}$, Li Qin ${ }^{\text {a }}$ \\ ${ }^{a}$ School of Machinery and Automation, Wuhan University of Science and Technology, Wuhan \\ 430081, China \\ ${ }^{\mathrm{b}}$ Intelligent Systems and Biomedical Robotics Group, University of Portsmouth, Portsmouth, PO1 \\ 3HE, UK
}

\begin{abstract}
Fiber Bragg grating (FBG) based force sensing technology receives much attention from various research communities in robotics. In this paper, we investigate a three-axis FBG force sensor for robot finger. A special separated elastic structure with six FBGs attached has been designed for three-axis force sensing. Strain distribution and dynamic performance of the proposed structure are simulated by using finite element analysis (FEA). Six FBGs with different wavelengths were arrayed along a single fiber and divided into three groups to measure $F_{x}, F_{y}$, and $F_{z}$, respectively. The experimental results demonstrate that the sensor possesses good linearity, weak coupling, and creep resistance.
\end{abstract}

Keywords: Fiber Bragg grating (FBG); force sensor; multi-component force sensor; robot.

\section{INTRODUCTION}

Multi-axial force sensor is one of the most important external sensors for robot, which is essential to compliance control, force/position control, remote control and other feedback applications[1]. The development of multi-axial force sensors has been described and investigated for decades since 1970s, with the background that force information is crucial to the rapid development of intelligent robot.

It is well known that the elastic structure body and sensitive element are the two key components for a multi-axial force sensor, which are determinant to sensor capability. For elastic structure body, cross beams typed sensing element [2-4] and its evolution type $[5,6]$ are most widely used in commercial products and research 
reports because of their good symmetry, high degree of integration, and easy to be expanded. Parallel mechanisms, especially Stewart platform, are also widely adopted in multi-axis force/torque sensors, for its distinguishing advantages of high rigidity, symmetry and stress decoupling [7-9]. There are also other kinds of elastic structure for sensing element, such as E-type membrane [10, 11], ring-type [12], MEMS [13], and so on. For the sensitive element which sensing the deformation of elastic structure body, the traditional resistance strain gages are most commonly used, although it is prone to electromagnetic interference, tedious wires jointing, and zero temperature drift.

Recent years, fiber optic sensors offer a promising alternative to electric measurement elements. As a particular class of optical fiber sensors, fiber Bragg grating (FBG) sensors have attracted considerable attention from various research communities because of their distinguishing advantages, such as immunity to electromagnetic noise and optical power fluctuations, small profile, light weight, high resistance to hostile environments, no zero temperature drift and the fact that multiple FBGs can be arrayed along a single fiber [14-16].

In the past two decades, FBG sensors have been developed rapidly for practical use in civil structural health monitoring[17], mechanical equipment[18], aerospace engineering[19], and biomedicine[20]. In recent years, this advanced sensing technology has also been adopted in robotics, especially for robot force sensing and feedback [21, 22]. Several FBG-based Multi-axial force sensors have been proposed based on the structure of the classical cross beams and Stewart platform. Fernandez et al. developed a three component force sensor with eight multiplexed FBGs by using a Maltese cross shaped beams, which was recognized as the first time that multi-component force sensors based on multiplexed FBGs can be constructed[23]. Yong-Lae Park et al. embedded five FBGs in a robot exoskeletal finger for accurate two component force sensing and estimation of contact locations [24]. Mueller et al. proposed a FBG force/torque sensor with six degrees of freedom based on an elastic structure resembles the traditional Stewart platform. Six FBGs along a single fiber were twined on the elastic structure to detect 6-D force/torque, however, serious 
coupling exists between the 6-D outputs[25]. Jiang Qi et al. developed a three dimensional force sensor based on a structure consists of three beams with FBG attached, and coupling also exists between the 3-D outputs [26].

With the rapid development of minimally invasive robotic surgery (MIRS), effective force sensing and feedback technology of operating forceps or needle, which is crucial to successful rate of operation, has been investigated by many research communities. FBG becomes the preferred sensing element for the features of small size, immunity to electromagnetic interference, and physical robustness. Hoseok Song et al. developed a 3-axis force/torque sensing operating forceps. Four FBG strain sensors and four temperature compensation FBGs were used to measure $F_{z}, M_{x}$, and $\mathrm{M}_{\mathrm{y}}[27,28]$. Haslinger et al. designed a 6-D force/torque sensor for integration in instruments for MIRS. The mechanical structure of the sensor is similar to the Stewart Platform and six FBGs have been embedded inside the links [29]. Elayaperumal et al. developed a magnetic resonance imaging (MRI) compatible biopsy needle with FBGs for measuring interaction force and bending deflection of the needle as it is inserted into tissues[30, 31]. Russell Taylor et al. developed a 2-D and 3-D force sensing micro-forceps for robot assisted retinal microsurgery [32-35]. Three FBGs were longitudinally attached along the forceps shaft with 120 intervals to detect 2-D force, and one FBG was placed in the center of the tubular shaft to detect axial force. Similar to this measuring principle, Gijbels et al. designed a 2-D force sensing needle with three FBGs integrated into the tool shaft [36].

Currently, with the actual demand of society and economy development, special robot becomes an important development direction, such as patrol robot for EHV power line inspection, nuclear power station rescue robot, space robot etc. Severe environment conditions such as strong electromagnetic interference, nuclear radiation, space radiation, high and low temperature probably exist in the work environment of these robots, which made the conventional electric force sensors hard to work properly. And in some cases, only three dimensions force is needed, if a six-axis force/torque sensor is used to acquire the three-axis force information, it will result in a waste of resources, what's more, coupling influence among the six axes will 
degrade the measuring accuracy. Given these concerns, this paper introduces our work on an FBG-based three-axis force sensor for robot finger. A novel separated elastic structure has been proposed and validated by FEA. Six FBGs with different wavelengths were arranged into the elastic structure body, which could measuring $F_{x}$, $\mathrm{F}_{\mathrm{y}}$, and $\mathrm{F}_{\mathrm{z}}$ without dimension coupling. The mechanical structure design, measuring principle, and full-scale test have been presented.

The rest of the paper is organized as follows. In Section 2, the sensing principle of FBG is introduced first, and then a detailed introduction of the elastic structure design and FEA analysis are presented. Section 3 shows the FBG arrangement and measuring Principle. In Section 4, test and calibration results are described. Section 5 presents concluding remarks and ideas for future work.

\section{SENSOR DESIGN}

\subsection{FBG Working and Sensing Principle}

FBG is a periodic change of the refractive index in the core of a single mode optical fiber. As shown in Fig.1, when imported with a broadband light, the FBG reflects a specific narrowband range of the incident light. The central wavelength of the reflected light is just the Bragg wavelength $\lambda_{B}$, which can be described with the effective refraction index of the fiber core $n_{\text {eff }}$ and of the periodicity of the grating $\Lambda$ as[37]:

$$
\lambda_{B}=2 n_{e f f} \bullet \Lambda
$$

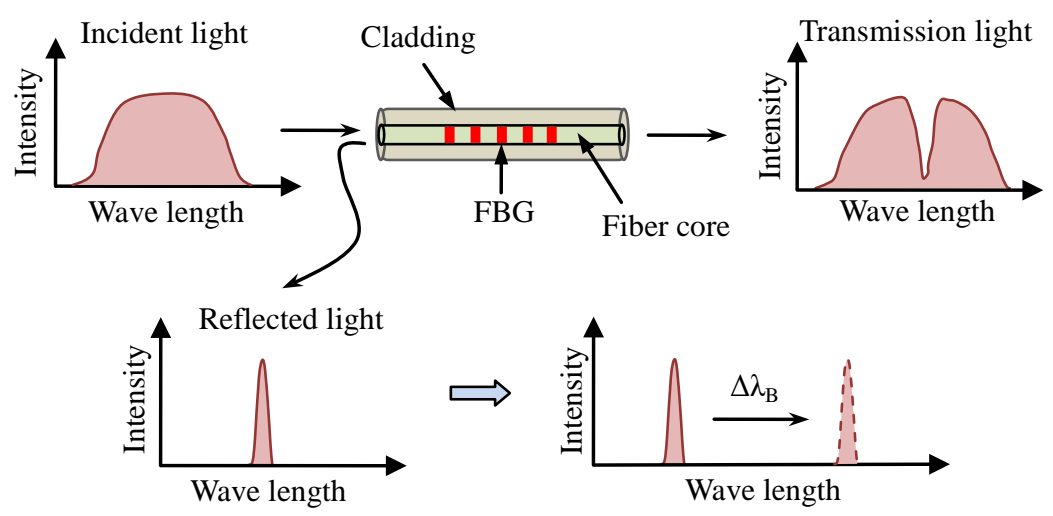

Fig. 1. Schematic diagram of working and sensing principle of FBG 
The wavelength shift of $\lambda_{B}$ is decided by the changes of $n_{\text {eff }}$ and $\Lambda$, which are mainly influenced by the axial strain $\Delta \varepsilon$ and environment temperature $\Delta \mathrm{T}$. The well known sensing equation of FBG is given by [38]:

$$
\frac{\Delta \lambda_{B}}{\lambda_{B}}=\left(\alpha_{f}+\xi\right) \Delta T+\left(1-P_{e}\right) \Delta \varepsilon
$$

Where $\lambda_{B}$ is the initial wavelength of FBG, $\alpha_{f}$ is the thermal expansion coefficient, $\xi$ is the thermal-optic coefficient and $P_{e}(\approx 0.22$ at room temperature $)$ is the effective photo-elastic coefficient.

\subsection{Mechanical Structure}

Fig.2 depicts the special elastic structure design of the proposed FBG force sensor. The elastic force-sensing element mainly includes an outer measuring body, an inner measuring body, a bottom cover, and the finger tip. Four vertical beams incorporated in the outer measuring body with 90 ० intervals are used as elastic deformation elements for a pair of orthogonal forces. The assembled sensor can be mounted using the four screw holes on the bottom off the outer measuring body. Top of the inner measuring body is a threaded bolt which is used to connect the finger tip. A cavity is designed in the upper part of the inner measuring body to receive one FBG, while a micro hole in the center is used to inject adhesive to fix the optical fiber. The lower part of the inner measuring body is a hollow thin-walled cylinder where two parallel slots with 90 intervals are cut out, which forms a flexibility for axial deformation. The bottom cover is designed as an accessory that plugged to the hole at the bottom of the inner measuring body. The micro hole at the center of the bottom cover is used to pass through and fix the optical fiber.

The following special connections are used within the assembly of the sensor. The bottom cover is inserted into the inner measuring body with an interference fit and laser spot welding. The same connection has been established between the base of the inner measuring body and the bottom hole of the outer measuring body. The assembly between the upper part of the inner measuring body and the upper part of the outer measuring body is a transition fit, which permits free sliding along the axial direction. 
Hence, axial forces form the finger tip are only transmitted to the inner measuring body, whereas radial forces are only transmitted from the finger tip to the outer measuring body.

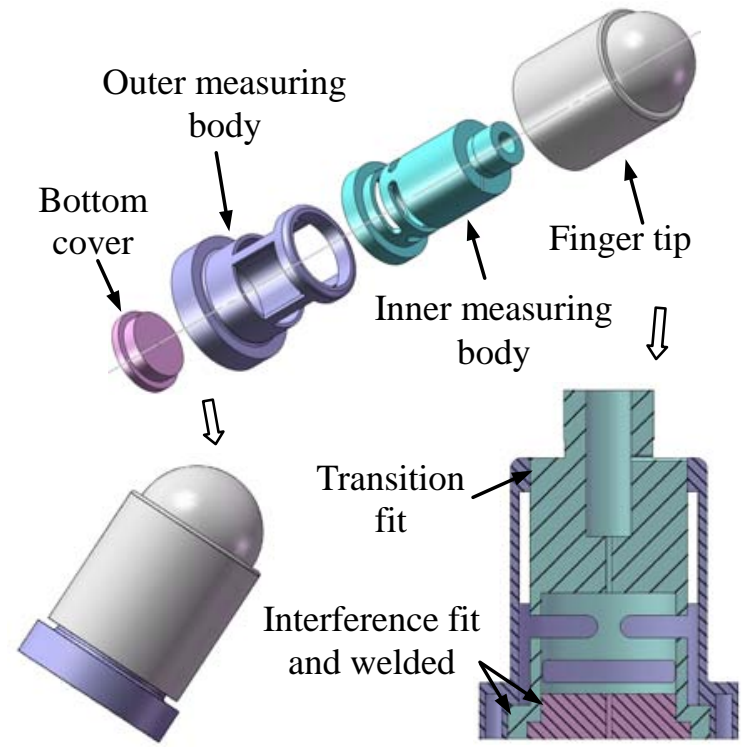

Fig. 2. Schematic of elastic structure for FBG three- axis force sensor

\subsection{Strain Distribution and Dynamic Analysis}

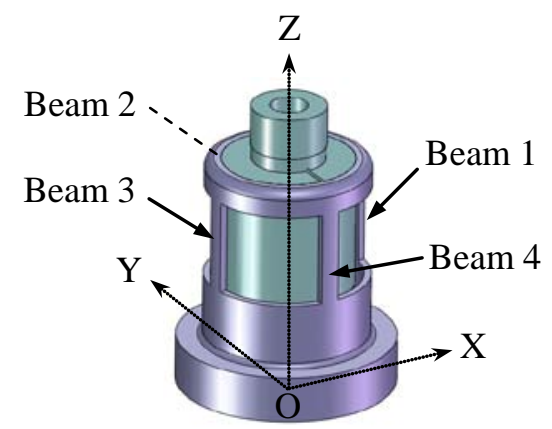

Fig. 3. Schematic of three-dimensional coordinate system

A three-dimensional coordinate system was established as shown in Fig.3. Four vertical beams of the outer measuring body were named as beam1, beam2, beam3 and beam4 in turn. The central axis of the elastic structure is $\mathrm{Z}$ axis. The center lines of beam 1 and beam 3 are located in plane XOZ, while beam 2 and beam 4 are located in plane YOZ. According to the initial design idea, beam1 4 are used to detect a pair of orthogonal forces. When $\mathrm{F}_{\mathrm{x}}$ acts on the threaded bolt of the inner measuring body, beam 1 and beam 3 bends and products surface strain, while beam 2 and beam 4 locate 
at the neutral layer and no surface strain is produced, and the result is inverted for the active force $F_{y}$. The central axis of the elastic structure always locates at the strain neutral layer, which makes it insensitive to $F_{x}$ and $F_{y}$, and that's why the inner measuring body is designed to detect the axial force $\left(\mathrm{F}_{\mathrm{z}}\right)$.

To verify the applicability of the proposed elastic structure, strain distribution analysis of the structure under force loading was simulated with the FEA software Solidworks simulation. During FEA, to simplify the structure, the screw threads at the top of inner measuring body and the bottom of outer measuring body are omitted, and the corresponding diameters are replaced by a crest diameter for the screw thread. The finger tip is not involved during analysis, and the material of the other components is aluminium alloy, and parameters used in the simulation are as follows: Young's modulus $E=6.9 \times 10^{10} \mathrm{~Pa}$, Poisson's ratio $\mu=0.33$.

Under the analysis of $F_{x}$, an applied force of $10 \mathrm{~N}$ acts on the top of inner measuring body. Based on the geometrical and measuring characteristic of FBG, FBGs should be attached on the surface of the beams along the center line (Fig.4-a). FBG mainly detects the surface strain along the $\mathrm{Z}$ axis. From the simulated strain distribution along $\mathrm{Z}$ axis (Fig.4-b), we discovered that the surface of beam1 and beam3 produce a clear strain.

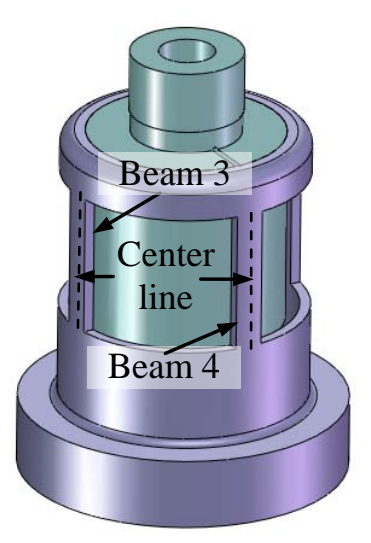

(a)

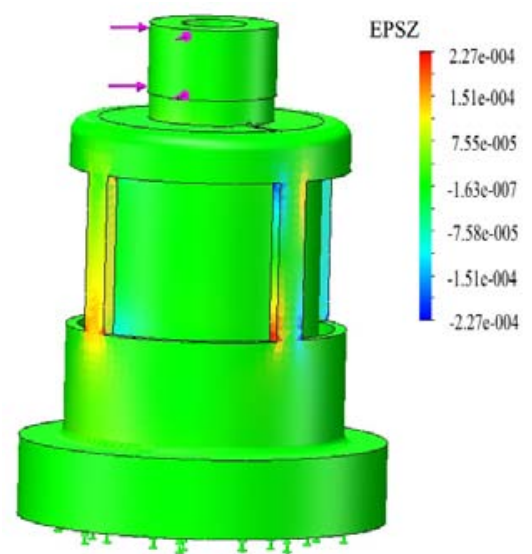

(b)

Fig. 4. (a) Schematic of center line of each beam; (b) Strain distributions on the elastic structure simulated by FEA

Detailed strain distribution tends along the center line of each beam have been investigated. Further FEA results displaying strain trends for FBGs along their 
detecting directions are shown in Fig.5. Fig.5(a) shows the strain trends curve along the center line from the bottom to the top of beam1 4. From the four strain trend curves, it is clear that the region near the bottom of beam3 produces maximum positive strain, and the region near the bottom of beam 1 produces maximum negative strain, while strain along the center line of beam 2 and beam 4 approximates to zero. Therefore, FBGs for orthogonal forces $\left(F_{x}\right.$ and $\left.F_{y}\right)$ measuring should be arranged on the surface near the bottom of each beam for maximum detection sensitivity (as marked in Fig.5-a).

The wavelength shift of FBG is influenced both by strain and temperature. Therefore, using the difference of the wavelength shift brought by the two positive and negative strains between the two opposite FBGs as the sensing signal, the effect of temperature cross-sensitivity could be avoided and the sensitivity can be improved (Fig.5-b).

When the elastic structure is under the active force $F_{y}$, the result is similar.

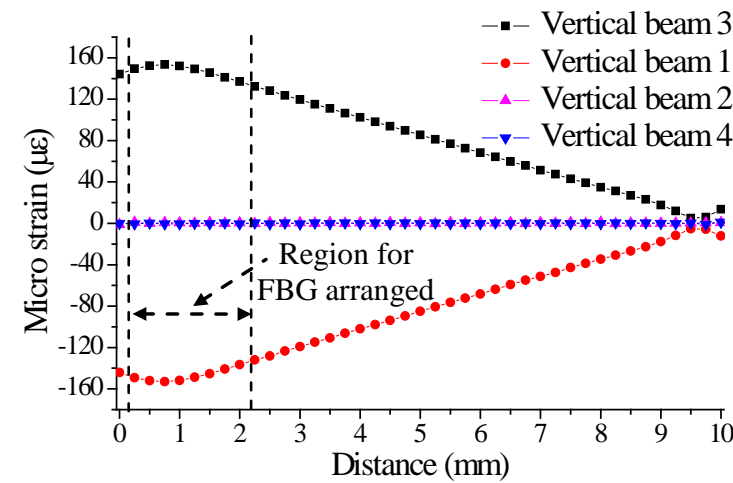

(a)

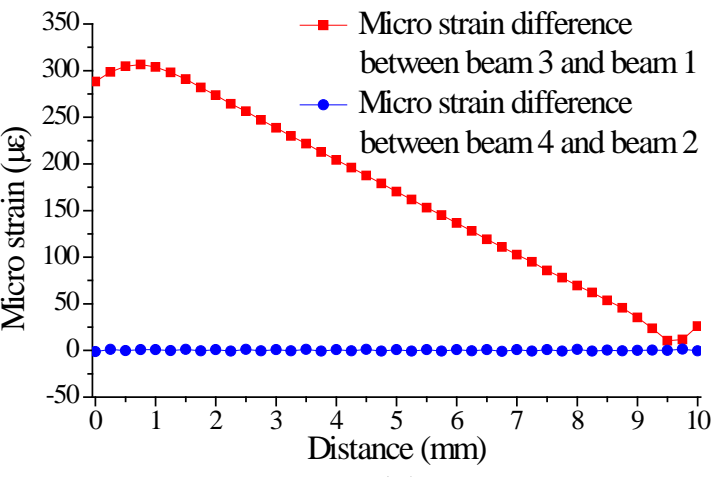

(b)

Fig. 5. (a) Strain distribution curves of the four beams; (b) Strain difference between the two opposite beams

Under the analysis of $F_{z}$, the outer measuring body has been removed for the reason that the inner measuring body could slide freely along $\mathrm{Z}$ axis and $\mathrm{F}_{\mathrm{Z}}$ only acts on the inner measuring body. A segment of optical fiber has been adopted and fixed on the micro holes of inner measuring body and bottom cover to serve as a FBG (Fig.6-a). The material of optical fiber is glass, and parameters used in the simulation are as follows: Young's modulus $E=5.5 \times 10^{10} \mathrm{~Pa}$, Poisson's ratio $\mu=0.25$, and the 
applied force $F_{z}=-5 \mathrm{~N}$. The simulated strain distribution along $\mathrm{Z}$ axis is shown in Fig.6 (b), obviously, deformation mainly occurs at the parallel slots and the optical fiber, which verifies the rationality of the FBG arrangement for measuring $\mathrm{F}_{\mathrm{z}}$.

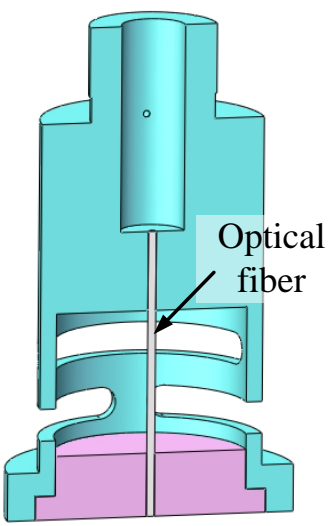

(a)

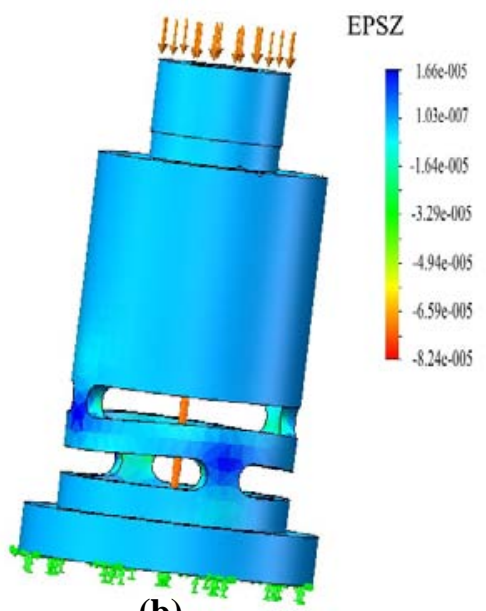

(b)

Fig. 6. (a) Assembly diagram of the optical fiber; (b) Strain distributions on the inner measuring body and optical fiber simulated by FEA

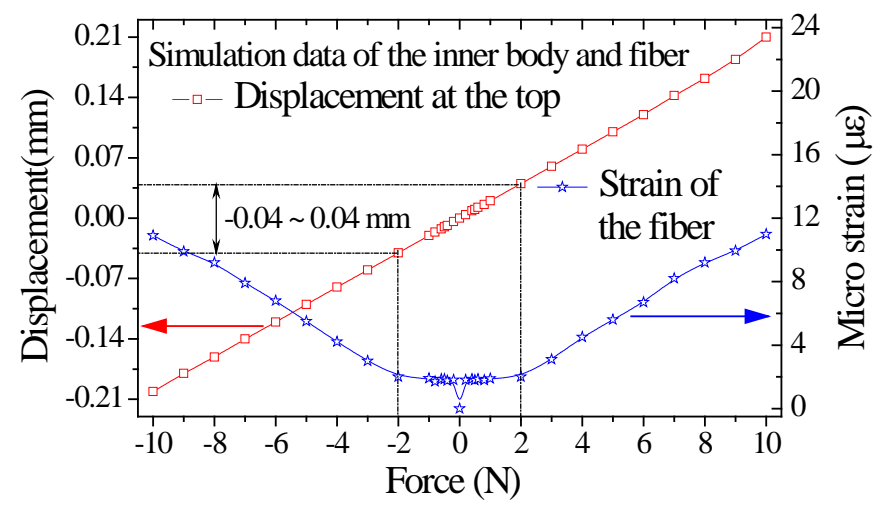

Fig. 7. Simulation results of the inner body and optical fiber

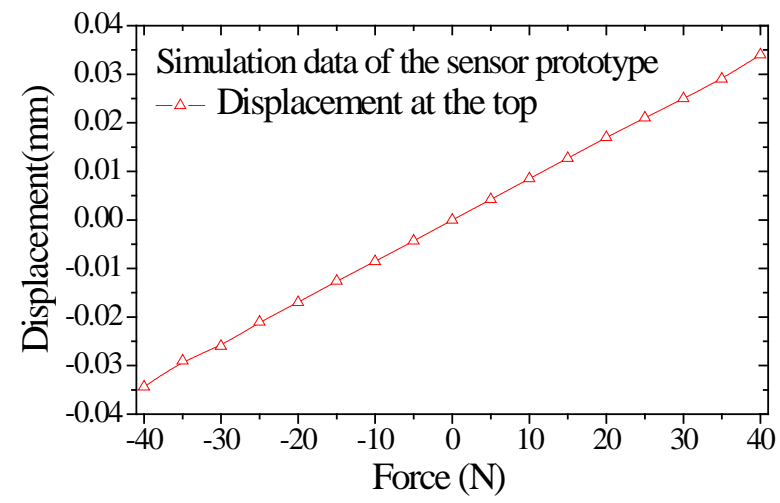

Fig. 8. Simulation results of the sensor prototype

Under large bending along the two-dimensional plane of $\mathrm{X}$ and $\mathrm{Y}$, optical fiber in 
the inner measuring body could shift away from the bending neutral surface and produce crosstalk strain[35]. In order to obtain the bending limit, a simulation of the inner body and optical fiber response under force loading has been carried out to analyze the crosstalk. As shown in Fig.7, we investigated the relationship between the deformation displacement at the top of the inner body and the strain produced by optical fiber under different values of Fx. We found that when the displacement over $0.04 \mathrm{~mm}$, the optical fiber starts to produce coupling strain. A simulation of the sensor prototype which includes the inner body and the outer body under force loading has also been carried out as shown in Fig.8. When the displacement at the top of the inner body reaches $0.04 \mathrm{~mm}$, an acting force over $40 \mathrm{~N}$ is needed, which means that the measuring range should no more than $40 \mathrm{~N}$.
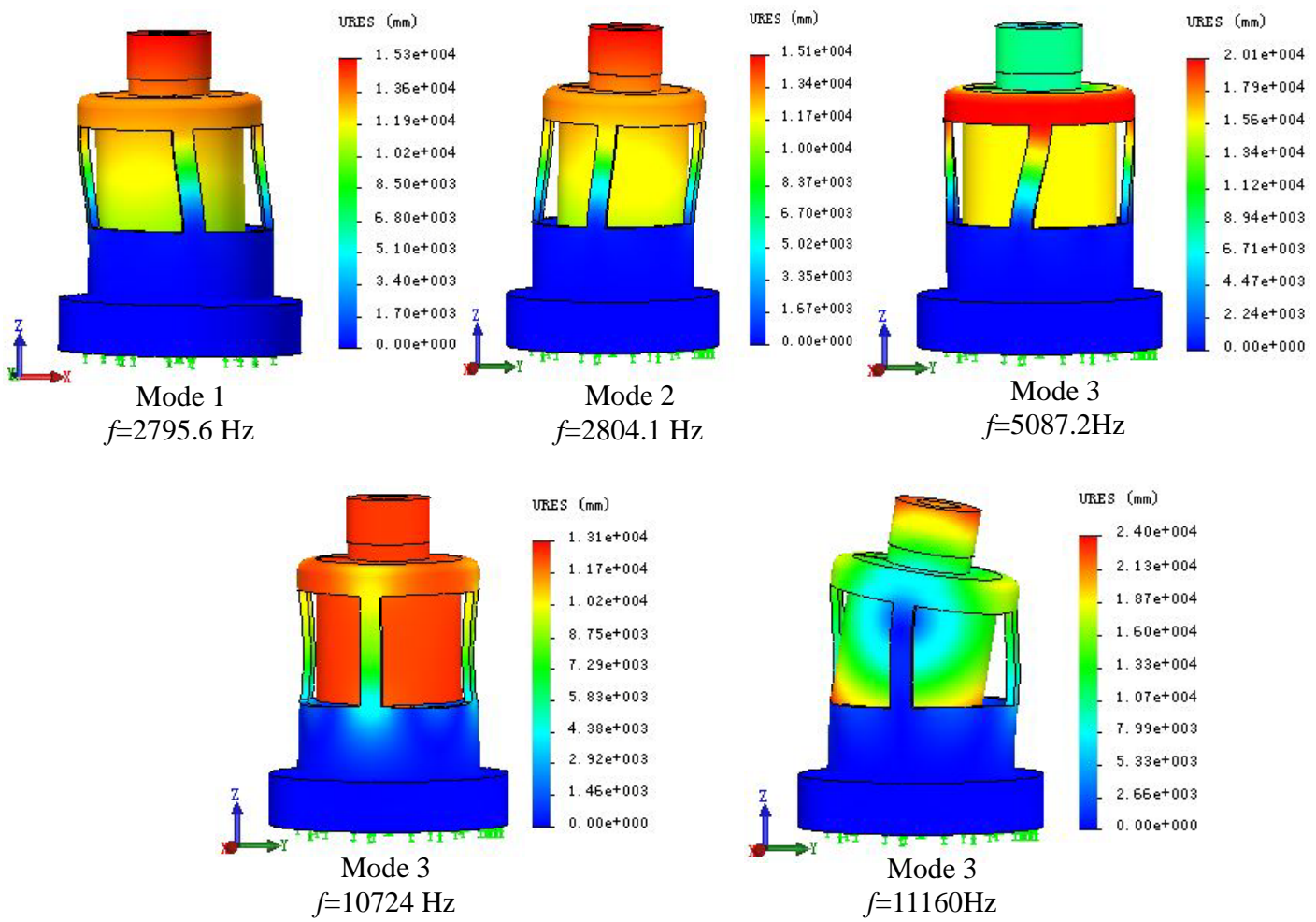

Fig. 9. The first 5 inherent frequencies and vibration modes of the elastic structure

As dynamic characteristics are important to formulate the scope of application of a sensor, we investigated the dynamic response of the elastic structure. Model analysis has been made to understand the first 5 inherent frequencies and vibration modes by Solidworks simulation. As shown in Fig.9, the first two vibration modes ( $f \approx 2800 \mathrm{~Hz})$ which corresponding to the orthogonal $\mathrm{X}$ axis and $\mathrm{Y}$ axis are dominant. Step response 
of the first two dominant vibration modes under a frequency sweep of $0-3000 \mathrm{~Hz}$ with a sinusoidal force of $5 \mathrm{~N}$ and a step of $10 \mathrm{~Hz}$ has been demonstrated in Fig. 10. The scope of the sensor could be $0-1867 \mathrm{~Hz}$, which is often determined to the two thirds of the first inherent frequency according to experience.

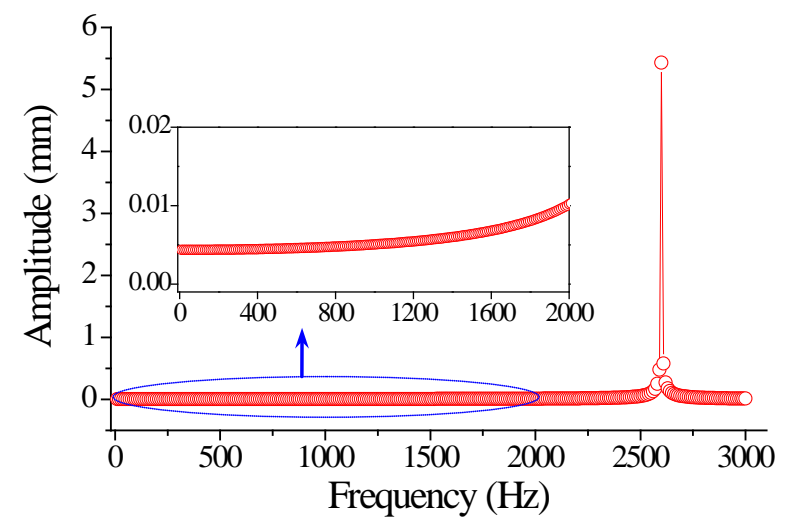

Fig. 10. Amplitude-frequency response of the first two dominant vibration modes

\section{FBG ARRANGEMENT}

Six FBGs with different reflection wavelengths have been fabricated at an interval about $3 \mathrm{~nm}$ along a single model optical fiber using phase mask technology without recoating, and the effective length of each FBG is about $2 \mathrm{~mm}$. Then, bare FBGs were attached to the sensor structure, whereas the coating layer of the fiber between FBGs is retained. Schematic diagrams of the arrangement for the six FBGs are shown in Fig.11. After fabricating, the initial wavelengths of FBG1 FBG6 in room temperature are $1552.151 \mathrm{~nm}, 1555.126 \mathrm{~nm}, 1558.055 \mathrm{~nm}, 1560.998 \mathrm{~nm}, 1563.986 \mathrm{~nm}$, and $1567.046 \mathrm{~nm}$, respectively.

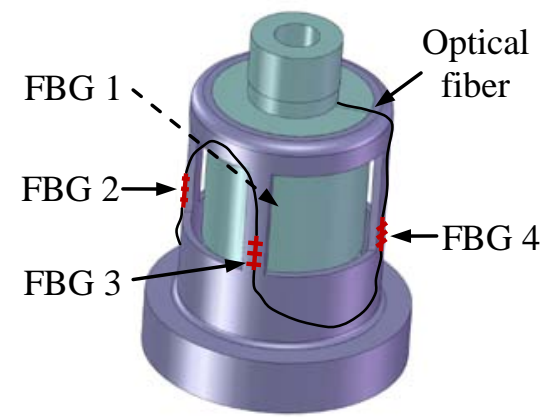

(a)

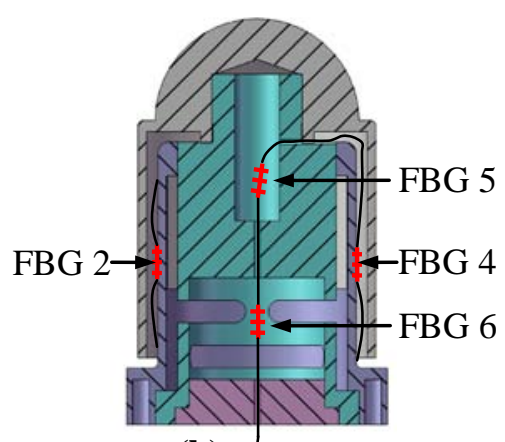

(b)

Fig. 11. (a) Schematic diagrams of the arrangement for FBG1 4; (b) arrangement for FBG5 and FBG6 
FBG1 FBG4 have been adhered on the surface near the bottom of beam1 beam 4 (as marked in Fig.5-a) using a commercial adhesive. As shown in Fig.11 (b), the optical fiber between FBG4 and FBG5 threads the minute-groove in the inner measuring body, and FBG5 is hanged freely in the cavity of the inner measuring body to serve as the temperature compensation FBG for FBG6. The fiber on either side of FBG6 has been fixed in the micro centre holes of the inner measuring body and the bottom cover. Additionally, FBG6 has been pre-stretched about $1.7 \mathrm{~nm}$ before being fixed so as to measuring the deformation along negative $\mathrm{Z}$ axis. The bending radius of the fiber between FBG1 4 is about 9 mm, and that between FBG4 and FBG5 is about $7.5 \mathrm{~mm}$. A comparison of the reflectance spectrum of the six FBGs before and after adhering on the sensor structure has been illustrated in Fig.12. Photos of the elastic structure with six FBGs arranged and prototype of the sensor have been presented in Fig.13 (a) and (b), respectively. The diameter of the sensor at finger tip is $\Phi 20 \mathrm{~mm}$, and the total height is $35.5 \mathrm{~mm}$.

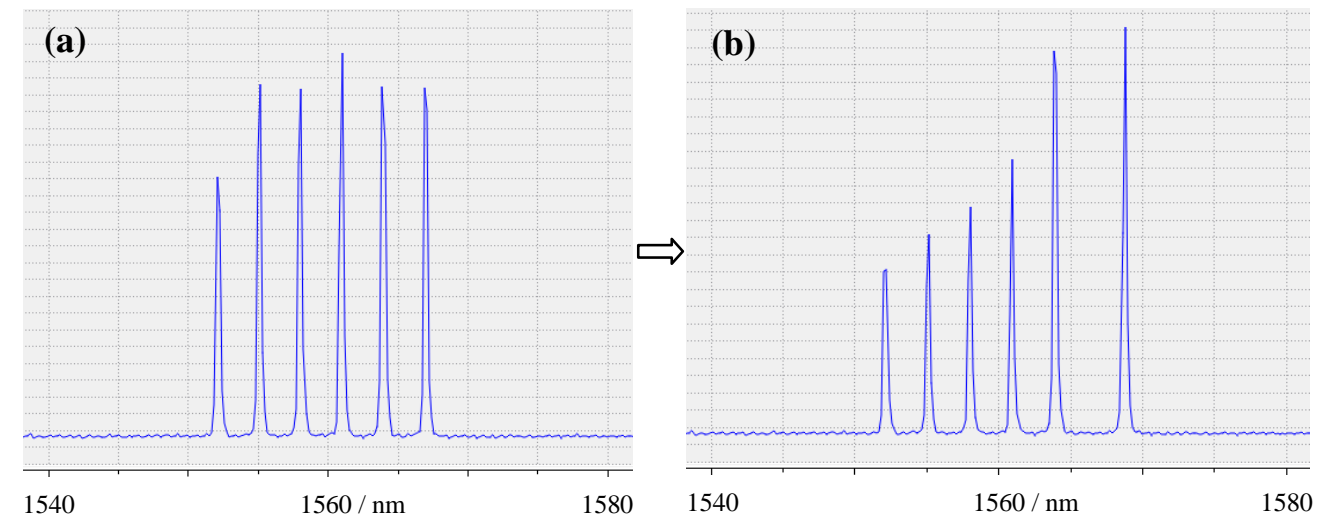

Fig. 12. Comparison of the reflectance spectrum of the six FBGs: (a) initial reflectance spectrum; (b) after adhering

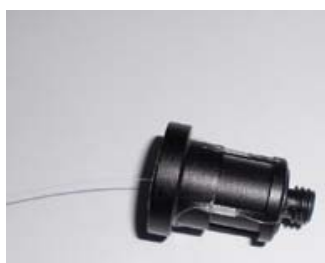

(a)

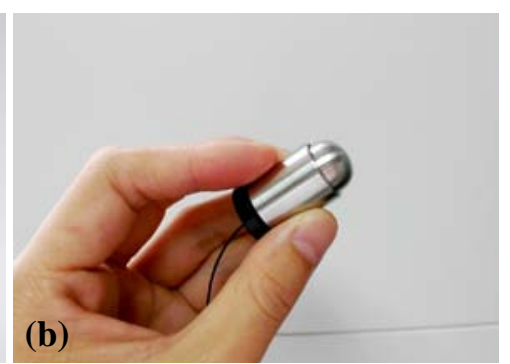

Fig. 13. photos: (a) the elastic structure with FBGs arranged; (b) prototype of the sensor 
The adhering methods of FBG1 FBG4 are same, so the wavelength shift of this four FBGs undergo temperature variation can be considered equal. Obviously, the pure strains on beam 1 and beam 3 that brought by $F_{x}$ is represented by the difference wavelength of FBG1 and FBG3: $\Delta \lambda_{3-1}=\Delta \lambda_{3}-\Delta \lambda_{1}$. In the same way, $\Delta \lambda_{4-2}=\Delta \lambda_{4}-\Delta \lambda_{2}$ represents $F_{\mathrm{y}}$. The adhering methods of FBG5 and FBG6, which are used to measure $\mathrm{F}_{\mathrm{z}}$, are different, so the temperature sensitivity of FBG5 and FBG6 are different. A coefficient $K=\mathrm{k}_{\mathrm{FBG} 6} / \mathrm{k}_{\mathrm{FBG5}}$ is introduced to represent the ratio of the temperature sensitivity between FBG5 and FBG6. So, the pure strains brought by $F_{z}$ is represented by $\Delta \lambda_{6-5}=\Delta \lambda_{6}-K \cdot \Delta \lambda_{5}$. The above is the measuring method for the three-axis force using the difference of the wavelength shift.

\section{EXPERIMENTAL RESULTS}

First, in order to obtain the value of temperature coefficient $K$ between FBG5 and FBG6 and investigate the sensor's temperature compensation performance, the temperature sensitivities of each FBG versus temperature increase have been tested. During the test, a thermostat is used to change the surrounding temperature of the sensor. The temperature is changed between $35{ }^{\circ} \mathrm{C}$ and $65{ }^{\circ} \mathrm{C}$ and the sampling interval is $10^{\circ} \mathrm{C}$. The temperature sensitivities of the six FBGs are listed in Fig.14. Obviously, sensitivities of FBG1 FBG4 are approximately equal, and the coefficient $K$ between FBG5 and FBG6 is $24.16 / 11.09 \approx 2.179$. Therefore, $\Delta \lambda_{3-1}=\Delta \lambda_{3}-\Delta \lambda_{1}$ is the pure wavelength shift differences for $F_{x}, \Delta \lambda_{4-2}=\Delta \lambda_{4}-\Delta \lambda_{2}$ for $F_{y}$, and $\Delta \lambda_{6-5}=\Delta \lambda_{6}-2.179 \cdot \Delta \lambda_{5}$ for $F_{\mathrm{z}}$.

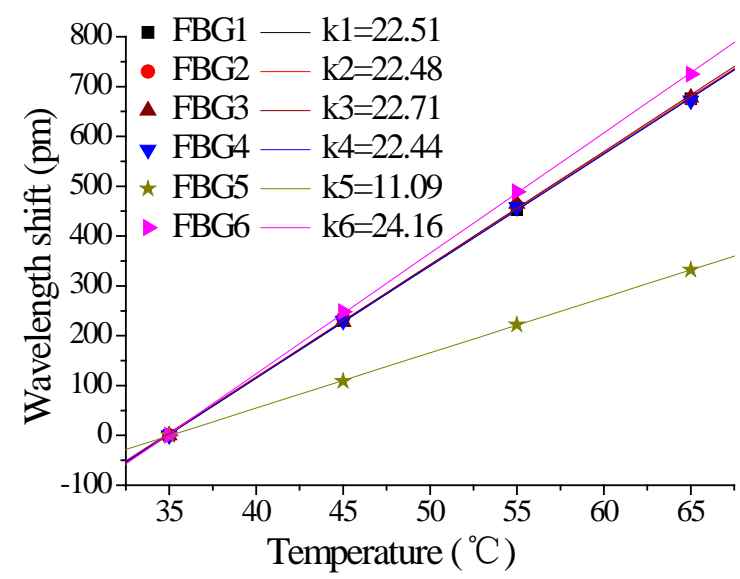


Fig. 14. The temperature sensitivities of each FBG

Subsequently, static calibration experiments have been implemented on the multi-dimension force/torque calibration platform, as shown in Fig.15. The sensor prototype is bolted on a lifting platform and the gravity of several standard weights is used as the force load which acts on the sensor prototype through a wire rope. Here the acceleration of gravity in our laboratory ( $30{ }^{\circ} \mathrm{N}, 114{ }^{\circ} \mathrm{E}$ ) equals $9.793 \mathrm{~m} / \mathrm{s}^{2}$. Considering the actual demand of our robot, we set the range of the force load within $25 \mathrm{~N}$. The force calibration begins by applying one of the three forces on the sensor with a series of standard weights from the minimum to the maximum. Meanwhile, the FBG wavelength data is recorded by a homemade FBG interrogator based on CCD spectrometer (acquisition frequency: $100 \mathrm{~Hz}$, accuracy: 2 pm, resolution: $0.1 \mathrm{pm}$ ).

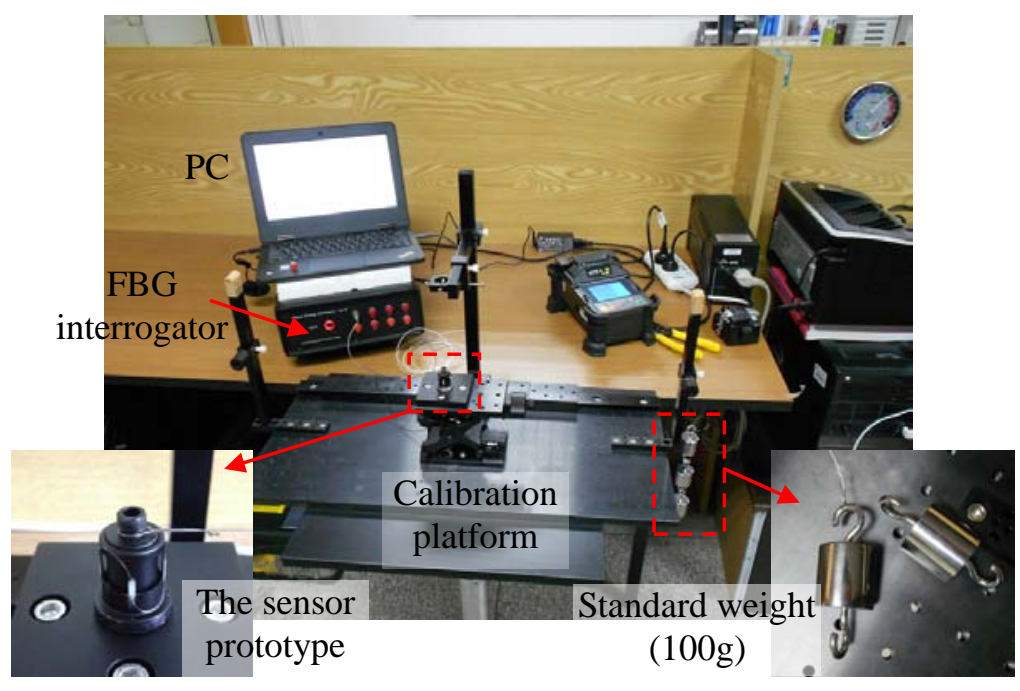

Fig. 15. Photo of the static calibration experiment

The relationship between wavelength outputs of each pair of FBGs and force load are shown in Fig.16(a, b and c), from which the three dimension coupling could be calculated as $0.823 \%, 0.974 \%$, and $0.677 \%$ for $\mathrm{F}_{\mathrm{x}}, \mathrm{F}_{\mathrm{y}}$, and $\mathrm{F}_{\mathrm{z}}$, respectively. The sensitivities of each measuring dimensionality are obtained from linear fit of the calibration data. As shown in Fig.16(d, e and f), it is clear that the sensor has good linear response in all three axis, and the sensitivity of $F_{x}, F_{y}$, and $F_{z}$ are $32.567 \mathrm{pm} / \mathrm{N}$, $33.97 \mathrm{pm} / \mathrm{N}$, and $44.116 \mathrm{pm} / \mathrm{N}$, respectively. By considering the FBG interrogator measuring accuracy of $2 \mathrm{pm}$, we can obtain that the sensor can measure the force with 
the minimum precision of $0.061 \mathrm{~N}, 0.059 \mathrm{~N}$, and $0.045 \mathrm{~N}$ for $\mathrm{F}_{\mathrm{x}}, \mathrm{F}_{\mathrm{y}}$, and $\mathrm{F}_{\mathrm{z}}$, respectively.

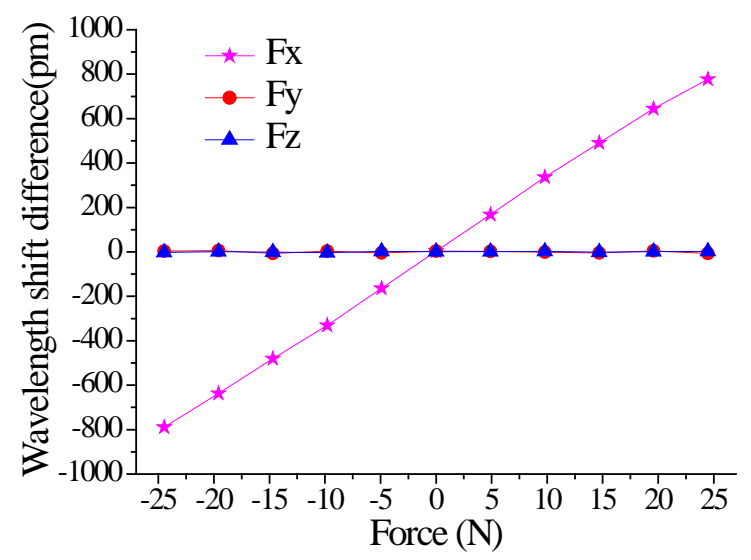

(a)

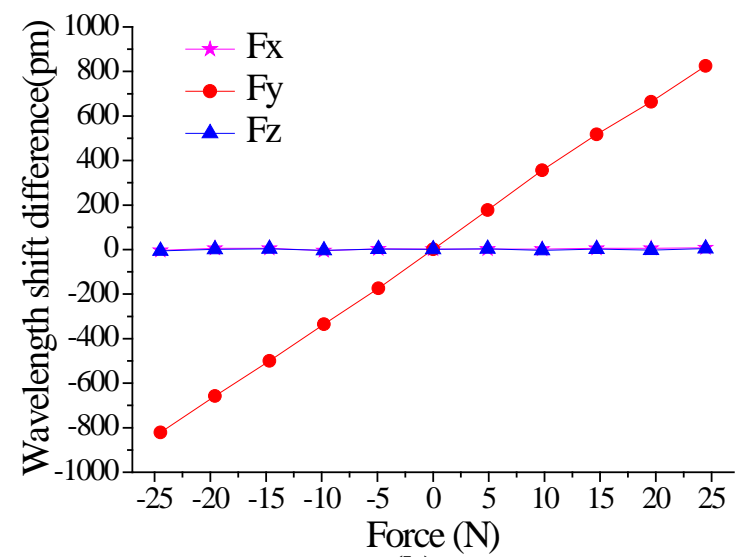

(b)

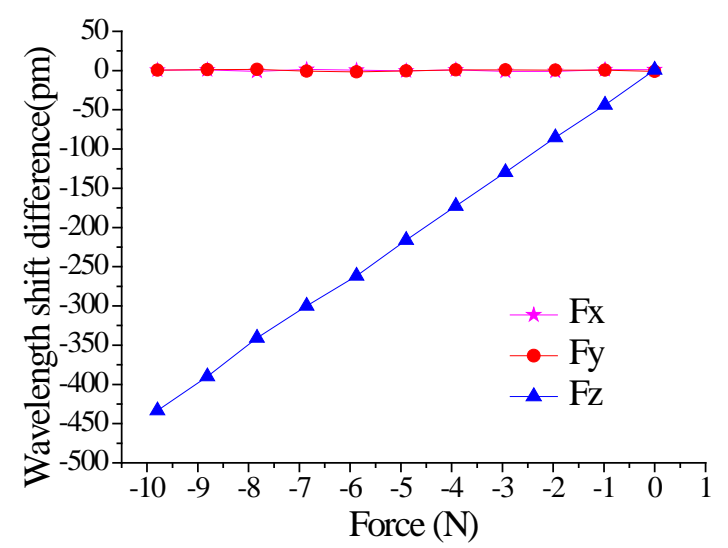

(c)

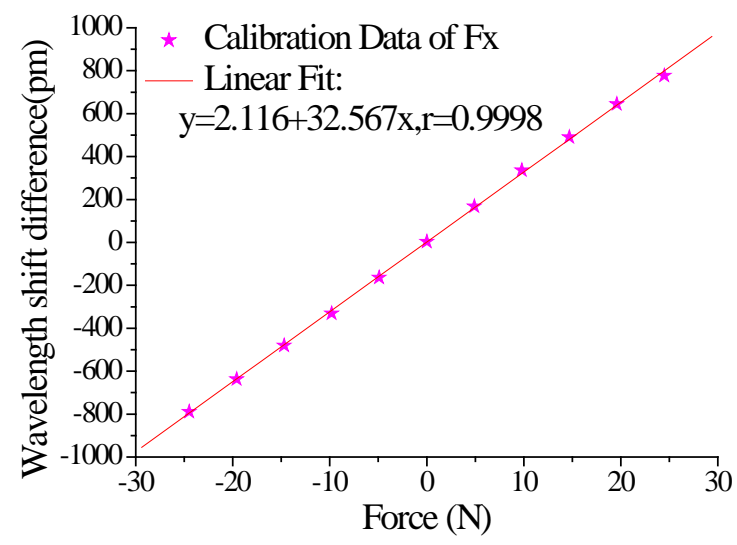

(d)

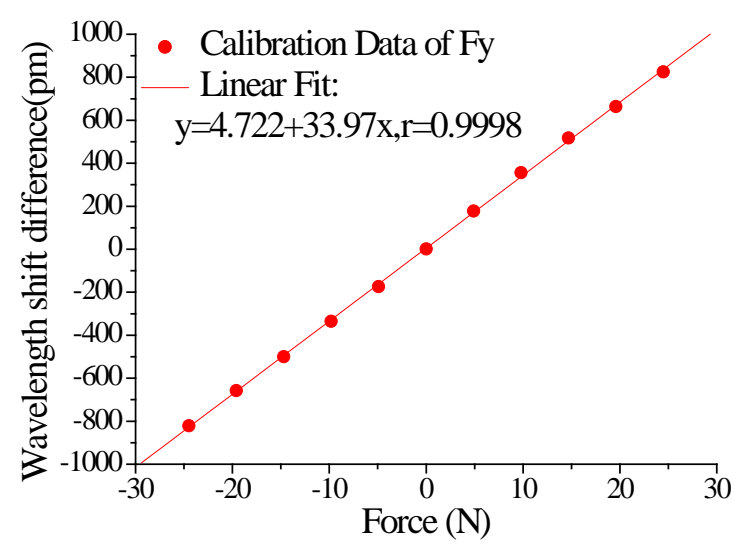

(e)

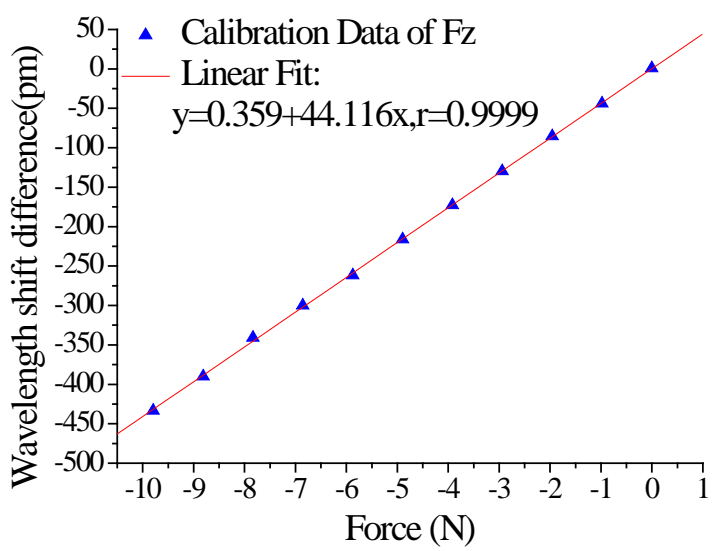

(f)

Fig. 16. Static calibration experiment results: (a) $\mathrm{F}_{\mathrm{x}}$; (b) $\mathrm{F}_{\mathrm{y}}$; (c) $\mathrm{F}_{\mathrm{z}}$; and linear fit results:

$$
\text { (d) } F_{x} \text {; (e) } F_{y} \text {; (f) } F_{z} \text {; }
$$




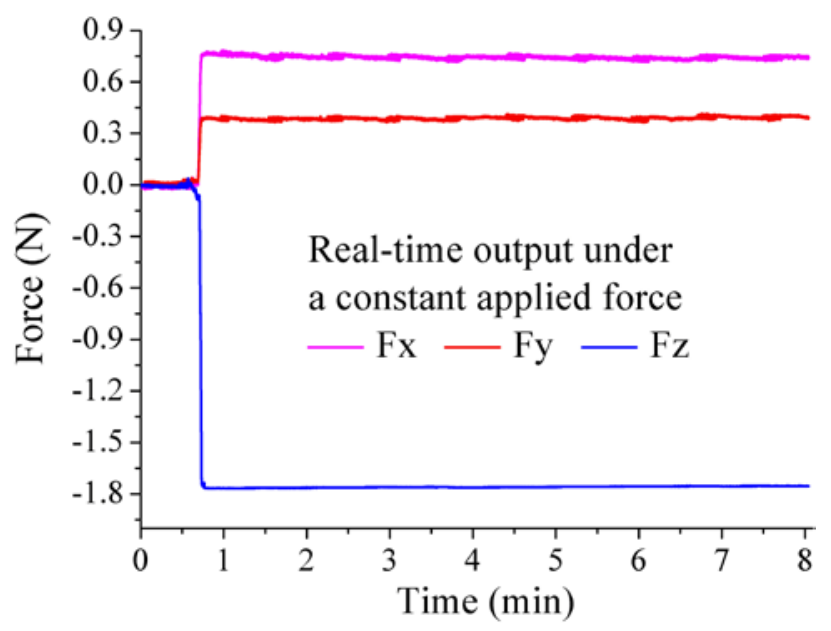

Fig. 17. Experiment results for creep resistance

Finally, for long term measurement, the creep behavior is needed for real-world applications. During this experiment, a constant load has been applied on the sensor over 7 minutes, and the output forces are shown in Fig17. As we can see, the real-time outputs of the three-axis force remain relatively unchanged, which demonstrates that the sensor has good creep resistance.

\section{CONCLUSION}

The work presented in this paper has described the development of an FBG-based three-axis force sensor for robot finger. The content is presented in the following sequence: elastic structure design, strain distribution and dynamic analysis, and calibration test. A separated mechanical structure and special assembly relationship have been proposed for three-axis force detect. Six FBGs with wavelength interval of $3 \mathrm{~nm}$ have been adhered on the mechanical structure and partitioned into three groups to measuring the three-axis force without coupling.

Calibration tests have been carried out in temperature characteristic, static force response, and creep. The three dimension couplings are $0.823 \%, 0.974 \%$, and $0.677 \%$ for $F_{x}, F_{y}$, and $F_{z}$, respectively, and the sensitivity of $F_{x}, F_{y}$, and $F_{z}$ are $32.567 \mathrm{pm} / \mathrm{N}$, $33.97 \mathrm{pm} / \mathrm{N}$, and $44.116 \mathrm{pm} / \mathrm{N}$, respectively. Experimental results show that the sensor demonstrates an extreme linear response versus applied force, weak inter-dimensional couplings and creep resistance, which is promising for applications 
in robot sensing, especially in the acquiring for tactile sense of robot manipulator. More testing work should be investigated in some complex and scurvy application environment in future to promote the performance of the FBG force fingertip sensor to product level.

\section{ACKNOWLEDGMENTS}

This work was financially supported by the Project of China Postdoctoral Science Foundation (No: 2015M572208), National Natural Science Foundation of China, NSFC (No: 51605348, 51575407, 51509194).

\section{References}

[1] Ge Yunjian, Zhang Jianjun, Ge Yu, et al. Ubiquitous sensing and robot perception[J]. ACTA AUTOMATICA SINICA, 2002, 28(S1): 125-133.

[2] Liang Q, Zhang D, Wang Y, et al. Development of a touch probe based on five-dimensional force/torque transducer for coordinate measuring machine $(\mathrm{CMM})[\mathrm{J}]$. Robotics and Computer-Integrated Manufacturing, 2012, 28(2): 238-244.

[3] Qiaokang Liang, Dan Zhang,Quanjun Song, et al. Design and fabrication of a six-dimensional wrist force/torque sensor based on E-type membranes compared to cross beams $[\mathrm{J}]$. Measurement, 2010, 43(10): 1702-1719.

[4] Shams S, Shin Dongik, Han Jungsoo, et al. Compact design of a torque sensor using optical technique and its fabrication for wearable and quadruped robots[C]. IEEE/RSJ International Conference on Intelligent Robots and Systems, 2011: 5127-5132.

[5] Zhang Weigang, Li Enbang, Xi Jiangtao, et al. Novel temperature-independent FBG-type force sensor[J]. Measurement science and technology, 2005, 16(8): 1600.

[6] Palli G, Moriello L, Scarcia U, et al. Development of an optoelectronic 6-axis force/torque sensor for robotic applications[J]. Sensors and Actuators A: Physical, 2014, 220: 333-346.

[7] Wang Zhijun, Yao Jiantao, Xu Yundou, et al. Hyperstatic analysis of a fully pre-stressed six-axis force/torque sensor[J]. Mechanism and Machine Theory, 2012, 57: 84-94.

[8] Baki P, Székely G, Kósa G. Design and characterization of a novel, robust, tri-axial force sensor[J]. Sensors and Actuators A: Physical, 2013, 192: 101-110. 
[9] ZhenYuan Jia, Sheng Lin, Wei Liu, Measurement method of six-axis load sharing based on the Stewart platform[J]. Measurment, 2010, 43(3):329-335.

[10] Qiaokang Liang, Dan Zhang,Yunjian Ge, et al. A novel miniature four-dimensional force/torque sensor with overload protection mechanism[J]. IEEE Sensors Journal, 2009, 9(12): 1741-1747.

[11] Qiaokang Liang, Dan Zhang, Song Quanjun, et al. A potential 4-D fingertip force sensor for an underwater robot manipulator[J]. IEEE Journal of Oceanic Engineering, , 2010, 35(3): 574-583.

[12] Sun Yongjun, Liu Yiwei, Jin Minghe, et al. Design and optimization of a novel six-axis force/torque sensor with good isotropy and high sensitivity[C]. IEEE International Conference on Robotics and Biomimetics, 2013: 631-638.

[13] Felix Beyeler, Simon Muntwyler, Bradley J. Nelson. A Six-Axis MEMS Force-Torque Sensor With Micro-Newton and Nano-Newtonmeter Resolution[J], Journal of Microelectromechanical Systems, 2009, 18(2): 433-441.

[14] Lin Yungbin, Chang Kuochun, Chern Jenn Chuan, et al. Packaging methods of fiber-Bragg grating sensors in civil structure applications[J]. IEEE Sensors Journal, 2005, 5(3): 419-424.

[15] Jiang H, Chen J, Liu T. Multi-objective design of an FBG sensor network using an improved Strength Pareto Evolutionary Algorithm[J]. Sensors and Actuators A: Physical, 2014, 220: 230-236.

[16] Li Xiong, Dongsheng Zhang, Litong Li, et al. EFPI-FBG hybrid sensor for simultaneous measurement of high temperature and large strain[J]. Chinese Optics Letters, 2014, 12(12): pp.120605

[17] Li X X, Ren W X, and Bi K M. FBG force-testing ring for bridge cable force monitoring and temperature compensation[J]. Sensors and Actuators A: Physical, 2015, 223: 105-113.

[18] Tianliang Li, Yuaegang Tan, Yi Liu, et al. A Fiber Bragg Grating Sensing Based Triaxial Vibration Sensor[J]. Sensors, 2015, 15(9): 24214-24229.

[19] Chen Ji-an, Huang Di, Zhao Haitao, et al. Fiber Bragg grating-based plane strain monitoring of aerostat envelope structures[J]. Applied optics, 2013, 52(19): 4631-4639.

[20] Nick J.vande Berg, Jenny Dankelman, John J.vanden Dobbelsteen. Design of an actively controlled steerable needle with tendon actuation and FBG-based shape sensing[J]. Medical 
engineering \& physics, 2015, 37(6): 617-622.

[21] Abushagur A. A. G, Arsad N, Reaz M. I, et al. Advances in bio-tactile sensors for minimally invasive surgery using the Fibre Bragg grating force sensor technique: A survey[J]. Sensors, 2014, 14(4): 6633-6665.

[22] Mishra V, Singh N, Tiwari U, et al. Fiber grating sensors in medicine: Current and emerging applications[J]. Sensors and Actuators A: Physical, 2011, 167(2): 279-290.

[23] A Fernandez Fernandez, F Berghmans, B Brichard, et al. Multi-component force sensor based on multiplexedfibre Bragg grating strain sensors[J]. Measurement science and technology, 2001, 12: 810-813.

[24] Yong-Lae Park, Seok Chang Ryu, Richard J. Black et al. Exoskeletal force-sensing end-effectors with embedded optical fiber-Bragg-grating sensors[J]. IEEE Transactions on Robotics, 2009, 25(6): 1319-1331.

[25] M. S. Mueller, L. Hoffmann, T. S. Buck, et al. Fiber Bragg grating-based force-torque sensor with six degrees of freedom[J]. International Journal of Optomechatronics, 2009, 3(3): 201-214.

[26] Jiang Qi, Song Jinxue, Gao Fangfang, et al. Research of robot multidimensional force sensing technology based on fiber Bragg grating[J]. Journal of optoelectronics laser, 2014, 25(11): 2123-2129.

[27] Song H, Kim H, Jeong J, et al. Development of FBG sensor system for force-feedback in minimally invasive robotic surgery[C]. 2011 Fifth International Conference on Sensing Technology. Palmerston North: IEEE, 2011: 16-20.

[28] Song H, Kim K, Lee J. Development of optical fiber Bragg grating force-reflection sensor system of medical application for safe minimally invasive robotic surgery[J]. Review of Scientific Instruments, 2011, 82(7): 074301.

[29] Haslinger R, Leyendecker P, Seibold U. A fiberoptic force-torque-sensor for minimally invasive robotic surgery[C]. IEEE International Conference on Robotics and Automation. Karlsruhe, Germany: IEEE, 2013: 4390-4395.

[30] Park Y L, Elayaperumal S, Daniel B, et al. Real-time estimation of 3-D needle shape and deflection for MRI-guided interventions[J]. IEEE/ASME Transactions on Mechatronics, 2010, 15(6): 906-915. 
[31] Elayaperumal S, Bae J H, Christensen D, et al. MR-compatible biopsy needle with enhanced tip force sensing[C]. IEEE World Haptics Conference (WHC). Daejeon, Korea: IEEE, 2013: 109-114.

[32] Iordachita I, Sun Z, Balicki M, et al. A sub-millimetric, $0.25 \mathrm{mN}$ resolution fully integrated fiber-optic force-sensing tool for retinal microsurgery[J]. International journal of computer assisted radiology and surgery, 2009, 4(4): 383-390.

[33] He X, Balicki M A, Kang J U, et al. Force sensing micro-forceps with integrated fiber Bragg grating for vitreoretinal surgery[C]. Optical Fibers and Sensors for Medical Diagnostics and Treatment Applications XII. San Francisco, California, USA: SPIE, 2012: 82180W-82180W7.

[34] Gonenc B, Handa J, Gehlbach P, et al. Design of 3-DOF force sensing micro-forceps for robot assisted vitreoretinal surgery[C]. 35th Annual International Conference of the IEEE Engineering in Medicine and Biology Society (EMBC). Osaka: IEEE, 2013: 5686-5689.

[35] He X, Handa J, Gehlbach P, et al. A submillimetric 3-dof force sensing instrument with integrated fiber bragg grating for retinal microsurgery[J]. IEEE Transactions on Biomedical Engineering, 2014, 61(2): 522-534.

[36] Gijbels A, Reynaerts D, Stalmans P, et al. Design and manufacturing of a 2-DOF force sensing needle for retinal surgery[C]. 4th Joint Workshop on New Technology for Computer/Robot Assisted Surgery. Genoa, Italy, 2014: 12-15.

[37] Kersey A D, Davis M A, Patrick H J, et al. Fiber grating sensors[J]. Journal of lightwave technology, 1997, 15(8): 1442-1463.

[38] Cheng J, Zhu W, Huang Z, et al. Experimental and simulation study on thermal gas flowmeter based on fiber Bragg grating coated with silver film[J]. Sensors and Actuators A: Physical, 2015, 228: 23-27.

\section{*Corresponding author:}

Yongxing Guo can be contacted at: yongxing_guo@wust.edu.cn 


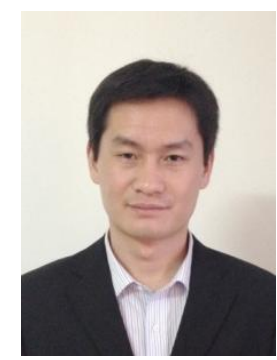

Yongxing Guo received the M. Eng and Ph. D. degrees in Measurement Control Technology and Instruments from National Engineering Laboratory for Fiber Optic Sensing Technology, Wuhan University of Technology, Wuhan China, in 2012 and 2014, respectively. He joined Wuhan University of Science \& Technology in 2014, as a postdoctoral research fellow and master's supervisor. He has authored or coauthored 16 journal and conference publications, and 4 issued Chinese patents. His current research interests include fiber Bragg grating sensing technology for special robot.

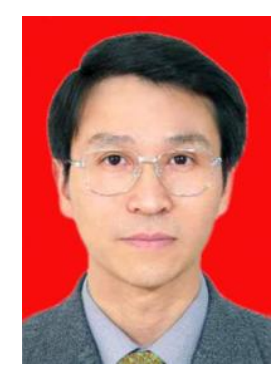

Jianyi Kong received the Ph.D. degree in mechanical engineering from Helmut Schmidt University, Germany, in 1994. He is currently a professor and doctoral supervisor of mechatronic engineering at Wuhan University of Science and Technology, Wuhan, PR China. He has authored or coauthored 200 journal and conference publications, and 6 academic monographs. His research interests include controlled mechanism, intelligent systems, and advanced measuring technology.

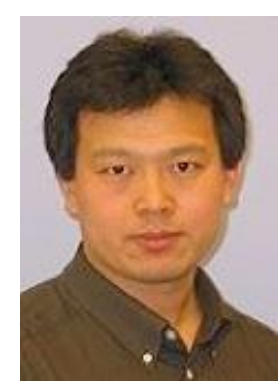

Honghai Liu received the Ph.D. degree in robotics from King's College London, London, U.K., in 2003. He is currently a professor of intelligent systems at the University of Portsmouth, Portsmouth, U.K. He previously held research appointments at the Universities of London and Aberdeen, and project Leader appointments in large-scale industrial control and system integration industry. His research interests include biomechatronics, pattern recognition, intelligent video analytics, intelligent robotics, robot sensing and their practical applications with an emphasis on approaches that could make contribution to the intelligent connection of perception to action using contextual information.

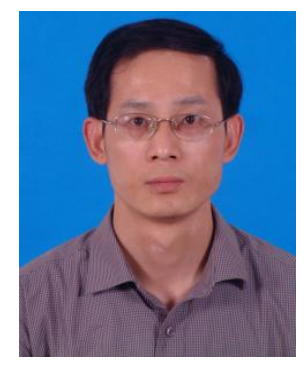

Hegen Xiong received the Ph.D. degree in mechanical engineering from Huazhong University of Science and Technology, in 2005. He is currently a professor and doctoral supervisor of mechanical engineering at Wuhan University of Science and Technology, Wuhan, PR China. He has authored or coauthored 70 journal and conference publications and 4 academic monographs. His research interests include intelligent robotics, robot sensing, $\mathrm{CAD} / \mathrm{CAM}$ and job shop scheduling. 


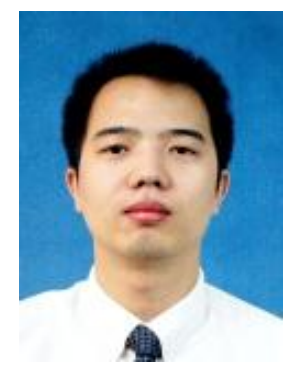

Gongfa $\mathbf{L i}$ received the Ph.D. degree in mechanical engineering from Wuhan University of Science and Technology, in 2008. He is currently a professor and master's supervisor of mechanical engineering at Wuhan University of Science and Technology, Wuhan, PR China. He has authored or coauthored 150 journal and conference publications and 10 issued Chinese patents. His research interests include intelligent design and control, intelligent robotics, and robot sensing.

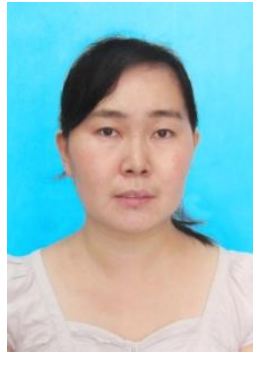

Li Qin received the $\mathrm{Ph}$. D. degree in energy and power engineering from Wuhan University of Technology, Wuhan China, in 2014, respectively. He joined Wuhan University of Science \& Technology in 2014, as a research assistant and master's supervisor. She has authored or coauthored 12 journal and conference publications, and 3 issued Chinese patents. Her current research interests include fiber Bragg grating based advanced measuring technology. 